\title{
OPERATIVE SKILL
}

\section{Is Pneumoperitoneum Really Required before Primary Trocar Insertion in Laparoscopy?}

\author{
Krishan Kapur \\ Senior Adviser (Obs and Gyne) and Endoscopic Surgeon, Command Hospital (Southern Command) \\ Pune, Maharashtra, India
}

Correspondence: Senior Adviser (Obs and Gyne) and Endoscopic Surgeon, Command Hospital (Southern Command) Pune-411040, Maharashtra, India

\begin{abstract}
Objectives: Traditionally a Veress needle is used to create a pneumoperitoneum before entering the primary trocar/cannula for laparoscopy. Insufflation of gas into the peritoneal cavity with the Veress needle is not without its problems. To do away with these problems the technique of direct trocar access was started.

Materials and methods: Between 01 Jan 2003 and 31 Dec 20082958 laparoscopic procedures were carried out in a Government hospital. 345 patients out of these had undergone previous laparotomies. In all cases direct trocar insertion without pneumoperitoneum was done.

Result: In this series there were no complications except in one case there was mesenteric injury with bleeding which required a laparotomy. There were no failed laparoscopies.

Conclusion: We have found that direct trocar insertion before creating pneumoperitoneum is a safe and effective method for laparoscopic access and offers advantages over the conventional access modality of using a Veress needle for pneumoperitoneum.
\end{abstract}

\section{INTRODUCTION}

Entering the abdomen is the most dangerous part of the laparoscopic procedure.

The traditional teaching for the performance of laparoscopy is to first introduce a Veress needle into the abdomen through an intraumbilical/subumbilical incision and insufflate gas so as to distend the abdomen and then to introduce the primary trocar and cannula. The long held perception is that after distension of the abdomen with gas the anterior abdominal wall moves away from vital organs thus making the entry of the thicker primary trocar into the abdomen a safer procedure with reduced incidence of bowel and vascular injury.

Insufflation of gas into the peritoneal cavity with the Veress needle is not without its problems like embolism, preperitoneal insufflation, failed pneumoperitoneum, bowel or vascular injury and the Veress needle procedure also takes more time. To do away with these problems the technique of direct trocar insertion into the peritoneal cavity without prior pneumoperitoneum was started.

\section{MATERIAL AND METHODS}

Between 01 Jan 2003 and 31 Dec 2008-2958 laparoscopic procedures were carried out in a Government hospital. 345 patients out of these had undergone previous laparotomies for either gynecological or surgical indication. In all patients direct trocar insertion without pneumoperitoneum was done, irrespective of the patients' age, weight or history of previous surgeries.

The technique followed was that a vertical intraumbilical incision was given (Fig. 1), the anterior abdominal wall was lifted with the left hand of the operator or if a grip of the anterior abdominal wall could not be obtained, help of a assistant was taken who lifted the abdominal wall to the right of the umbilicus (Fig. 2). In a few cases, where a proper grip of the anterior abdominal wall could not be obtained, two towel clips were used on either side of the umbilicus to lift the abdominal wall.

After the abdominal wall was lifted the primary trocar/ cannula with a pyramidal tip was inserted into the abdomen with a controlled but sustained pressure till two "gives" were felt. The first 'give' was on piercing the rectus sheath and the second 'give' on piercing the peritoneum (Fig. 3).

Once the trocar/cannula were felt to be in the abdomen the trocar was removed and the telescope introduced into the cannula to confirm the intraperitoneal placement as omentum/ gut could be visualized. After this gas was instilled into the abdomen to create a pneumoperitonium and the laparoscopy continued.

Of the 345 cases with previous laparotomies there were 23 cases where the umbilicus was involved in the primary surgical incision (vertical infraumbilical incision extending above the umbilicus, repair of umbilical hernia, etc). In these cases the primary trocar/cannula were inserted by the direct access method in the left upper quadrant of the abdomen at a point $3 \mathrm{~cm}$ below the left costal margin in the mid clavicular line.

\section{RESULT}

In our series there was no minor complications like gas embolism, subcutaneous emphysema. There was no case of bowel or 


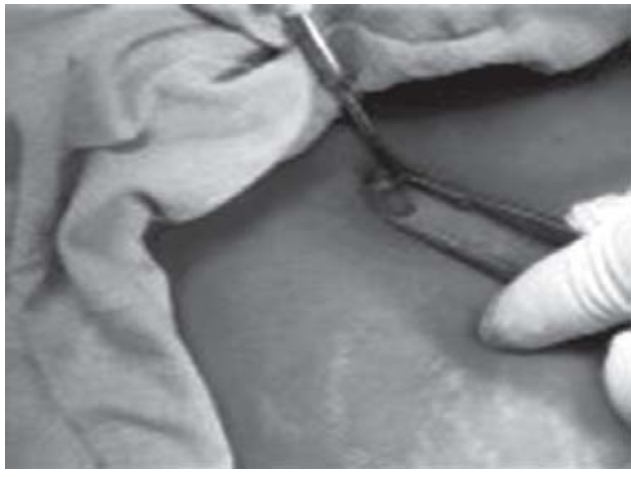

Fig. 1: Vertical intraumbilical incision

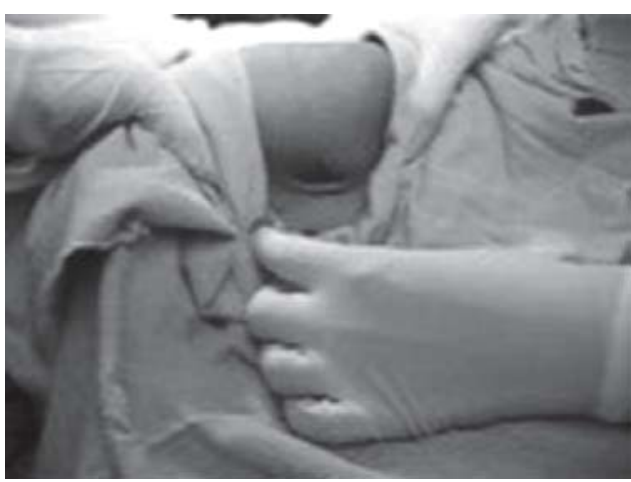

Fig. 2: Anterior abdominal wall lifted up

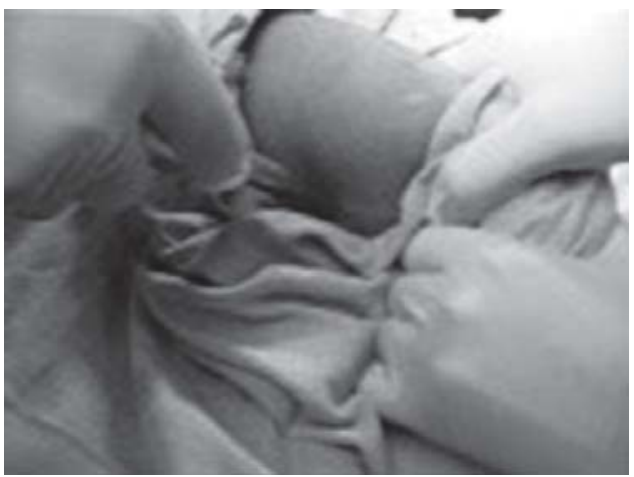

Fig. 3: Primary trocar/cannula being entered without pneumoperitoneum

vascular injury except in one case with no previous surgery the gut mesentry was injured resulting in intra-abdominal bleeding. As the bleeder could not be identified laparoscopically a laparotomy was done and the bleeding arrested.

In our series there were no failed laparoscopies and no case required more than 3 attempts to enter the abdomen.

\section{DISCUSSION}

One of the keys to safe laparoscopic surgery is an expeditious and reliable access to the abdomen. Abdominal access in endoscopic surgery carries with it a finite risk of bowel, vascular injury along with other complicatios like subcutaneous emphysema, failed laparoscopy. The techniques used to enter the abdomen are either by blind insertion of Veress needle to create pneumoperitoneum followed by the insertion of the primary trocar/cannula or by direct insertion of the trocar/ cannula without prior pneumoperitoneum or by open laparoscopy where the trocar and cannula are inserted after formally opening the abdomen. Most laparoscopic surgeons today use the Veress needle technique due to the long held perception that this technique reduces the incidence of visceral and vascular injuries. Review of the published literature demonstrated that bowel and vessel perforation rates (requiring laparotomy and resuscitation) are 1:1000 regardless of whether the method of gaining peritoneal access was open laparoscopy, Veress needle insufflation or direct trocar. ${ }^{1}$

The use of Veress needle to create pneumoperitoneum is associated with certain complications like gas embolism, subcutaneous emphysema, failed pneumoperitoneum, bowel insufflation. The incidence of these complications can be reduced by using the direct trocar insertion technique., 3

A number of studies have documented that direct trocar entry is safe and associated with lesser complications than abdominal access after use of Veress needle. ${ }^{4,5}$ In our study as well we find that direct trocar access is safe and effective. We had no minor complications and in only one case out of 2958 there was an injury to the gut mesentry requiring laparotomy. This injury occurred in a case with no risk factors but was performed by a surgeon new to the technique of direct access.

The biggest advantage of using direct trocar insertion is that the incidence of gas embolism is less as gas is insufflated only after intraperitoneal placement of the cannula has been confirmed. Direct trocar insertion also allows immediate recognition and rapid treatment of major blood vessel laceration and these points have been identified in reducing laparoscopy associated mortality. ${ }^{1}$ Direct trocar insertion also helps in reducing the time of surgery. ${ }^{2}$ Thus we see that direct trocar insertion scores over Veress needle insertion on many counts and we have had no problem in using this technique.

\section{CONCLUSION}

In this era of minimal access surgery, laparoscopic surgeons and gynecologists are looking for safe and effective laparoscopic access techniques. We have found that direct trocar insertion before creating pneumoperitoneum is a safe and effective method for laparoscopic access and offers 
advantages over the conventional access modality of using a Veress needle for pneumoperitoneum.

\section{REFERENCES}

1. Woolcot R. The safety of laparoscopy performed by direct trocar insertion and carbon dioxide insufflation under vision. Australian and NewZealand Journal of Obstetrics and Gynaecology 1997 May;37(2):216-19.

2. Byron JW, Markenson G, Miyazawa K. A randomized comparison of Veress needle and direct trocar insertion for laparoscopy. Surgery, Gynecology and Obstetrics 1993 Sept; 177(3):259-62.
3. Hill DJ, Maher PJ. Direct cannula entry for laparoscopy. Journal of the American Association of Gynecologic Laparoscopists 1996 Nov;4(1):77-79.

4. Jacobson MT, Osios J, Bizhang R, et al. The direct trocar technique: An alternative approach to abdominal entry for laparoscopy. Journal of Society of Laparoendoscopic Surgeons 2002 Apr-June;6(2):169-74.

5. Molloy D, Kalloo PD, Cooper M, Nguyen TV. Laparoscopic entry: A literature review and analysis of techniques and complications of primary port entry. Australian and New Zealand Journal of Obstetrics and Gynaecology 2002 Aug;42(3): 246-54. 